University of Nebraska - Lincoln

DigitalCommons@University of Nebraska - Lincoln

Faculty Publications from the Department of Electrical \& Computer Engineering, Department Electrical and Computer Engineering

2011

\title{
Transparent interconnections formed by rapid single-step fabrication of graphene patterns
}

\author{
J. B. Park \\ University of Nebraska-Lincoln \\ W. Xiong \\ University of Nebraska-Lincoln \\ Z. Q. Xie \\ University of Nebraska-Lincoln \\ Y. Gao \\ University of Nebraska-Lincoln \\ M. Qian \\ University of Nebraska-Lincoln
}

See next page for additional authors

Follow this and additional works at: https://digitalcommons.unl.edu/electricalengineeringfacpub

Part of the Computer Engineering Commons, and the Electrical and Computer Engineering Commons

Park, J. B.; Xiong, W.; Xie, Z. Q.; Gao, Y.; Qian, M.; Mitchell, M.; Mahjouri-Samani, M.; Zhou, Y. S.; Jiang, L.; and Lu, Yongfeng, "Transparent interconnections formed by rapid single-step fabrication of graphene patterns" (2011). Faculty Publications from the Department of Electrical and Computer Engineering. 222. https://digitalcommons.unl.edu/electricalengineeringfacpub/222

This Article is brought to you for free and open access by the Electrical \& Computer Engineering, Department of at DigitalCommons@University of Nebraska - Lincoln. It has been accepted for inclusion in Faculty Publications from the Department of Electrical and Computer Engineering by an authorized administrator of DigitalCommons@University of Nebraska - Lincoln. 


\section{Authors}

J. B. Park, W. Xiong, Z. Q. Xie, Y. Gao, M. Qian, M. Mitchell, M. Mahjouri-Samani, Y. S. Zhou, L. Jiang, and Yongfeng Lu 


\title{
Transparent interconnections formed by rapid single-step fabrication of graphene patterns
}

\author{
J. B. Park, ${ }^{1}$ W. Xiong, ${ }^{1}$ Z. Q. Xie, ${ }^{1}$ Y. Gao, ${ }^{1}$ M. Qian, ${ }^{1}$ M. Mitchell, ${ }^{1}$ M. Mahjouri-Samani, ${ }^{1}$ \\ Y. S. Zhou, ${ }^{1}$ L. Jiang, ${ }^{2}$ and Y. F. Lu ${ }^{1, a)}$ \\ ${ }^{1}$ Department of Electrical Engineering, University of Nebraska-Lincoln, Lincoln, Nebraska 68588-0511, USA \\ ${ }^{2}$ Department of Mechanical and Automation Engineering, Beijing Institute of Technology, Beijing 100081, \\ People's Republic of China
}

(Received 2 June 2011; accepted 13 July 2011; published online 1 August 2011)

\begin{abstract}
We developed a process to form transparent interconnections using graphene patterns that were synthesized by laser chemical vapor deposition. The number of graphene layers was tightly controlled by laser scan speed. Graphene patterns were fabricated at a high scan speed of up to 200 $\mu \mathrm{m} / \mathrm{s}$ with a single-step process. The process time is about a million times faster than the conventional chemical vapor deposition method. The fabricated graphene patterns on nickel foils were directly transferred to desired positions on patterned electrodes. The position-controlled transfer with rapid single-step fabrication of graphene patterns provides an innovative pathway for graphene-based interconnections. (C) 2011 American Institute of Physics. [doi:10.1063/1.3622660]
\end{abstract}

Transparent interconnections are essential for fabricating transparent devices such as solar cells, touch sensors, and flat panel displays. Indium tin oxide (ITO) is widely used for transparent interconnections due to its easy manipulation and simple patterning processes. However, the main element of ITO, indium, is a limited source in earth and it cannot be used for flexible transparent interconnections due to its poor mechanical properties. The outstanding optical, ${ }^{1}$ mechani$\mathrm{cal}^{2}{ }^{2}$ and electrical ${ }^{3}$ properties of graphene make it attractive for applications in flexible transparent interconnections. ${ }^{4}$ Recent advances in large-scale synthesis of graphene films by chemical vapor deposition (CVD) on Ni (Ref. 5) and $\mathrm{Cu}$ (Ref. 6) layers are expected to enable various flexible transparent electronics. ${ }^{7}$ Large-scale graphene patterns can be achieved by a catalytic patterning process or direct patterning of graphene sheets for various applications, such as transparent interconnections for graphene-based devices. ${ }^{8}$

However, conventional CVD methods consist of several steps, including annealing, growth, cooling, and patterning processes for fabricating graphene patterns. The growth process needs a long processing time of up to $\sim 2 \mathrm{~h}^{5-7}$ The patterning process also requires expensive masks which is not cost effective. In addition to the lengthy and multistep processing time, the fabricated graphene sheet or patterned graphene also needs to be transferred onto nonconductive substrates for device applications. ${ }^{9}$ Position-controlled patterning processes based on lithography techniques have been applied for graphene interconnections. ${ }^{8}$ The lithography-based "top-down" approach is not only time consuming due to a multistep process but it also causes unavoidable contamination due to the strong dipole interactions between chemical groups and graphene during the patterning process. ${ }^{10}$ Moreover, the top-down approaches also cannot be directly applied to the interconnections among existing electrodes or for repairing graphene-based devices. Therefore, the transfer of graphene patterns directly onto desired positions of patterned electrodes (bottom-up) is effective partic-

\footnotetext{
${ }^{\text {a) }}$ Author to whom correspondence should be addressed. Electronic mail: ylu2@unl.edu. Tel.: (402) 472-8323.Fax: (402) 472-4732.
}

ularly for transparent interconnections on existing devices. Furthermore, the rapid fabrication of the graphene patterns also reduces overall processing time and the number of steps. However, an effective method for the position-controlled transfer of graphene patterns directly onto prepatterned electrodes has not been established. Fabrication of simple graphene patterns by conventional CVD methods is also time consuming and costly.

In this article, we present a simple process for fabricating transparent interconnections using graphene patterns that are fabricated by laser chemical vapor deposition (LCVD) ${ }^{11}$ under ambient pressure. The number of graphene layers fabricated on nickel foils was controlled by process parameters including laser scan speed and gas pressure. It was demonstrated that the rapidly fabricated graphene patterns on nickel foils were directly transferred onto the desired positions of prepatterned electrodes for transparent interconnections. We also measured the electrical resistivity of the graphene interconnections.

In the experiment, a thin nickel foil in a gas mixture of $\mathrm{CH}_{4}$ and $\mathrm{H}_{2}$ was irradiated by laser to induce a local temperature rise, thereby allowing the growth of graphene patterns on the substrate at room temperature. Thin nickel foils with a thickness of $0.025 \mathrm{~mm}$ were used as substrates. The fabrication of graphene patterns was conducted in a small vacuum chamber. A focused laser beam (532 nm) was used to increase the local temperature on the nickel foils in the chamber. The pressure of the chamber was kept at $\sim 700$ torr during the experiments. The gas ratio of $\mathrm{CH}_{4}: \mathrm{H}_{2}$ was optimized to $5: 2$. The laser power was controlled at about $5 \mathrm{~W}$ to induce the local temperature rise required for graphene growth. The laser beam was focused to about $20 \mu \mathrm{m}$. The scan speed of the motorized stage was controlled up to $\sim 200 \mu \mathrm{m} / \mathrm{s}$ to fabricate the graphene patterns.

To form graphene interconnections among electrodes, position-controlled transfer was carried out using a wet transfer procedure. The graphene pattern on a nickel foil was spin-coated with PMMA at $2500 \mathrm{rpm}$. The nickel foil was then wet etched using a commercially available etchant (Transience Co.) for $\sim 30 \mathrm{~min}$, resulting in a graphene pattern/PMMA film floating on the surface of the etchant. This 
film was then collected and rinsed in deionized (DI) water before transferring it onto a substrate with patterned electrodes. During the graphene pattern/PMMA transfer, the position of the graphene pattern/PMMA film on patterned electrodes can be easily controlled precisely under a microscope due to the thin water layer between the PMMA layer (hydrophobic surface) and prepatterned electrodes. The thin water layer between the PMMA and the prepatterned electrode was removed, and the electrode was dried after positioning the graphene pattern. Finally, the graphene PMMA layer on the patterned electrode was removed, allowing graphene patterns to connect the prepatterned electrodes.

Figure 1 shows the structural information of a typical graphene pattern on a nickel foil, which was fabricated by rapid single-step LCVD. Figure 1(a) shows an optical micrograph of a graphene pattern. It is not easy to distinguish between the graphene pattern and the $\mathrm{Ni}$ foil, because the graphene pattern is highly transparent. The typical Raman spectrum of graphene patterns in Fig. 2(b) clearly shows the evidence of a single-layer graphene structure. Both the single Lorentzian shaped 2D-band $\left(2690 \mathrm{~cm}^{-1}\right)$ with a full width at half maximum (FWHM) of $\sim 30 \mathrm{~cm}^{-1}$ and the high ratio of the peak intensities of 2D- to G-bands $\left(1582 \mathrm{~cm}^{-1}\right)($ ratio $=3-4)$ confirm the presence of a single-layer graphene pattern. ${ }^{5-7}$ The Raman mappings of the insets in Fig. 1(b) reveal that the intensities of the G- and 2D-band signals are clearly distinguished from the surrounding nonirradiated areas, thus demonstrating the controlled locality of the graphene pattern in the LCVD process. The Raman intensity distributions of the graphene patterns are relatively uniform in the laser-irradiated areas, also demonstrating the uniformity of the fabricated graphene patterns. The SEM image in Fig. 1(c) shows the surface morphology of the graphene sheet. We can clearly see the crumpled graphene sheet in a magnified inset image caused by the difference between the thermal expansion coefficients of nickel and graphene. Atomic force microscope (AFM) image in Fig. 1(d) also clearly shows the crumpled graphene sheet. The measured height of the ripples was about $\sim 30 \mathrm{~nm}$.

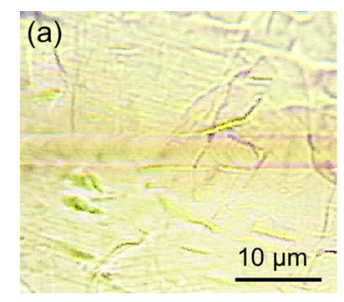

(c)
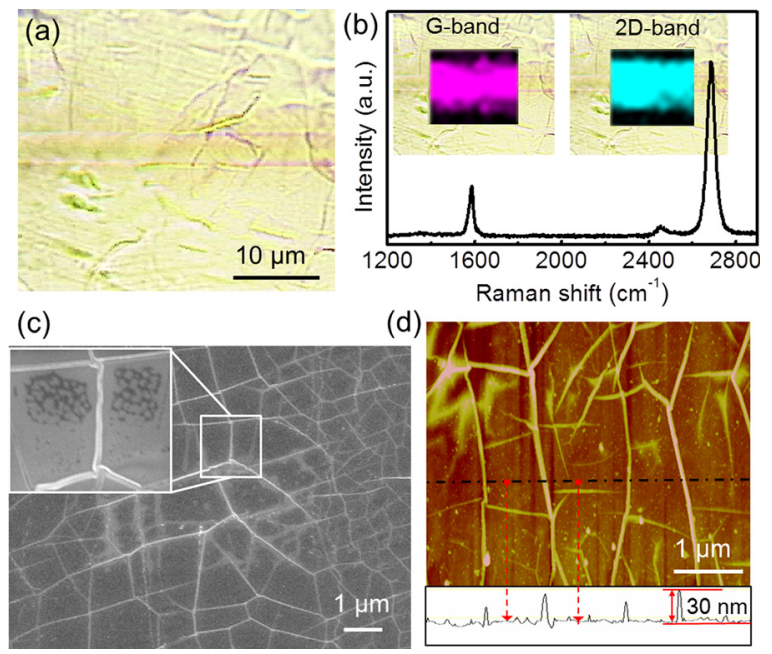

(d)

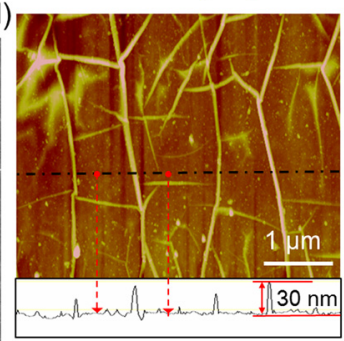

FIG. 1. (Color online) (a) Optical micrograph of a graphene pattern on a nickel foil and (b) its typical Raman spectrum at the center region. The insets show the Raman intensity mappings of G-band $\left(1582 \mathrm{~cm}^{-1}\right)$ and 2Dband $\left(2691 \mathrm{~cm}^{-1}\right)$ (c) SEM image of a graphene sheet inside of the graphene pattern. The inset shows the magnified image of crumpled graphene. (d) AFM image of the crumpled graphene.

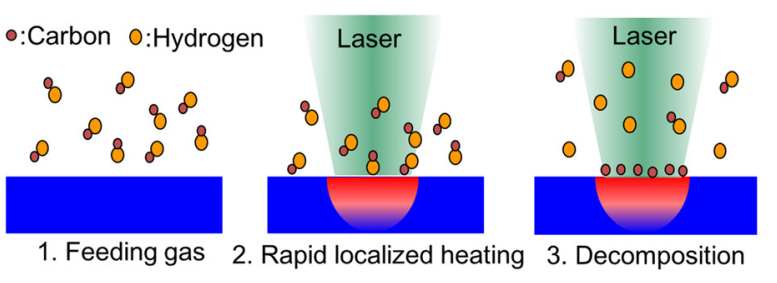

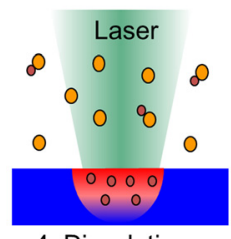

4. Dissolution

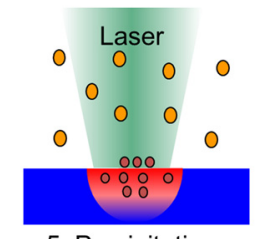

5. Precipitation

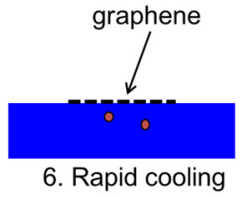

FIG. 2. (Color online) Illustration of rapid graphene growth through laserassisted vapor-liquid-solid model.

It is believed that these ripples make the graphene patterns more stable against mechanical stretching (more expandable) to graphene patterns. ${ }^{5}$

Surprisingly, the graphene patterns were fabricated with a rapid single-step process on a nickel foil without annealing, or patterning procedures. The pattern of $10 \mu \mathrm{m}$ width was directly fabricated at a high scan speed of $\sim 200 \mu \mathrm{m} / \mathrm{s}$ on the nickel foil. The graphene pattern with a size of $10 \times 10 \mu \mathrm{m}^{2}$ can be rapidly fabricated in $0.05 \mathrm{~s}$. It means that the growth process is up to a million times faster than that of conventional thermal CVD methods that take up to $\sim 2 \mathrm{~h}$ due to the slow heating and cooling rate with a multistep process. ${ }^{5,8}$ Figure 2 shows an illustration of graphene growth using the rapid single-step LCVD process. Unlike the conventional CVD method, reaction gases, including $\mathrm{CH}_{4}$ and $\mathrm{H}_{2}$, were fed into a vacuum chamber before heating the nickel foil (step 1). A focused laser beam irradiates on the nickel substrate to rapidly increase the local temperature through an inelastic electronphonon coupling ( $\sim 1$ ps, (Ref. 12)) (step 2). Rapidly heated nickel foil quickly decomposes the gaseous precursors on the local area of the nickel foil (step 3). At the same time, the decomposed carbon atoms dissolve into the nickel foil, and some of them are precipitated on the nickel foil (steps 4 and 5). Finally, the thin carbon layers are formed on the nickel foil by the rapid cooling process through fast thermal conduction after laser irradiation (step 6). The laser assisted vapor-liquidsolid process can be done between approximately several tens of picoseconds and several nanoseconds,,${ }^{13,14}$ meaning that the process time is about a million times faster than that of the conventional CVD method.

We studied the dependence of graphene growth on processing parameters including gas pressures and scan speeds. The number of graphene layers can be tightly controlled by the scan speed of the focused laser beam. Figures 3(a)-3(d) show the optical micrographs of the graphene patterns on a $\mathrm{SiO}_{2} / \mathrm{Si}$ substrates with respect to different scan speeds. A single-layer graphene pattern was obtained at a high scan speed $(200 \mu \mathrm{m} / \mathrm{s})$ in an ambient pressure (700 torr), as shown in Fig. 3(d). A double-layer graphene pattern was fabricated at a speed slightly lower than that used in fabricating the singlelayer graphene pattern, as shown in Fig. 3(c). Multi-layer graphene patterns start to form at about $170 \mu \mathrm{m} / \mathrm{s}$, as shown in Fig. 3(b). The number of graphene layers in patterns increases 

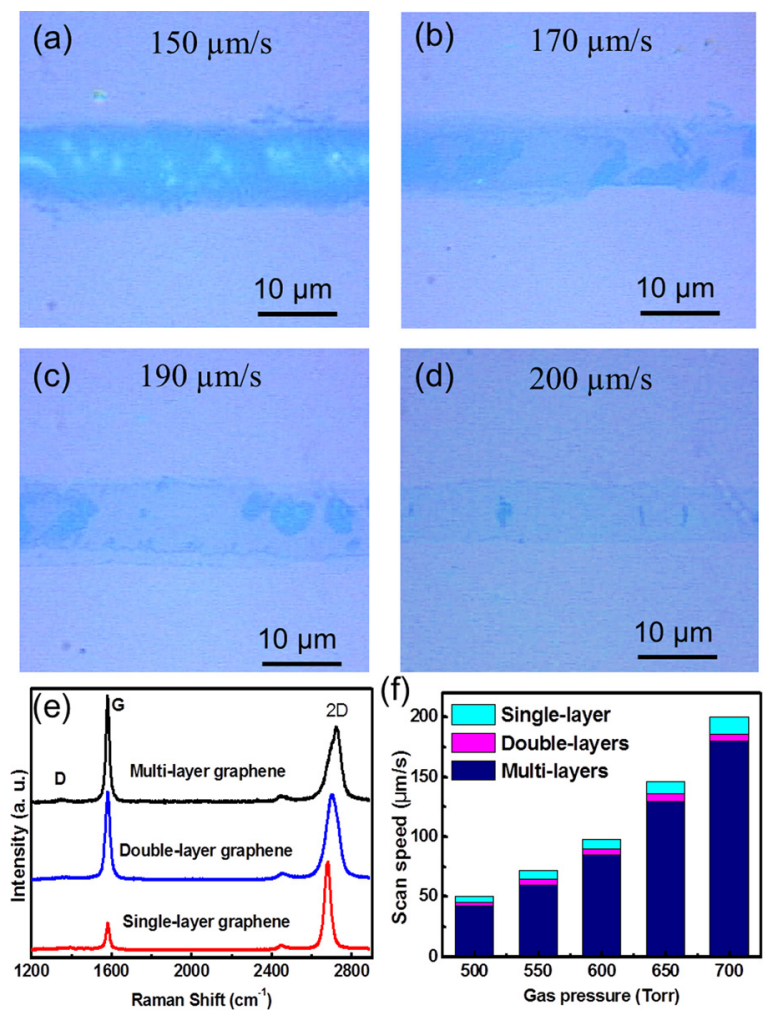

FIG. 3. (Color online) Optical micrographs of transferred (a) and (b) multilayer, (c) double-layer, and (d) single-layer graphene patterns on a $\mathrm{SiO}_{2} / \mathrm{Si}$ wafer $\left(\mathrm{SiO}_{2}=300 \mathrm{~nm}\right)$. (e) Typical Raman spectra of graphene patterns for multi-layer, double-layer, and single-layer graphene patterns. (f) Fabrication window of different layers of graphene pattern with respect to the gas pressures and scan speeds.

with decreasing scan speeds (Fig. 3(a)). Figure 3(e) shows the Raman spectra of different layers of graphene patterns that were fabricated at different scan speeds. The absence of Dband in the spectrum also confirms that a high-quality structure of graphene patterns was obtained. Figure 3(f) shows the control of graphene layers with respect to the gas pressures and scan speeds.

For the rapid interconnection process, the fabricated graphene patterns were directly transferred onto a prepatterned electrode. An optical microscope image in Fig. 4(a) shows a representative graphene pattern transferred onto gold electrodes. The graphene pattern was transferred onto the desired position of patterned electrodes through the position-controlled transfer technique. We can see the graphene pattern on the $\mathrm{SiO}_{2} / \mathrm{Si}$ substrate, but it is hard to see the graphene pattern on gold electrodes because single-layer graphene is highly transparent $(>97 \%){ }^{15}$ The sheet resistance of a single-layer graphene pattern was measured at $700 \Omega / \square$. Figure 3(c) and the inset, respectively, show a SEM image and an optical microscope image of a multi-layer graphene pattern on gold electrodes. We can still see the gold electrode through the multi-layer graphene pattern. Finally, we also measured the sheet resistance of multi-layer graphene pattern with respect to the scan speed as shown in Fig. 4(d). The sheet resistance can be reduced to below $\sim 120 \Omega / \square$ with $75 \%$ transparency $(514 \mathrm{~nm}$ ), which can be applied for transparent interconnections.

In conclusion, we have developed a method for rapid transparent interconnection that was supported by rapid single-step fabrication of graphene patterns. The laser-assisted
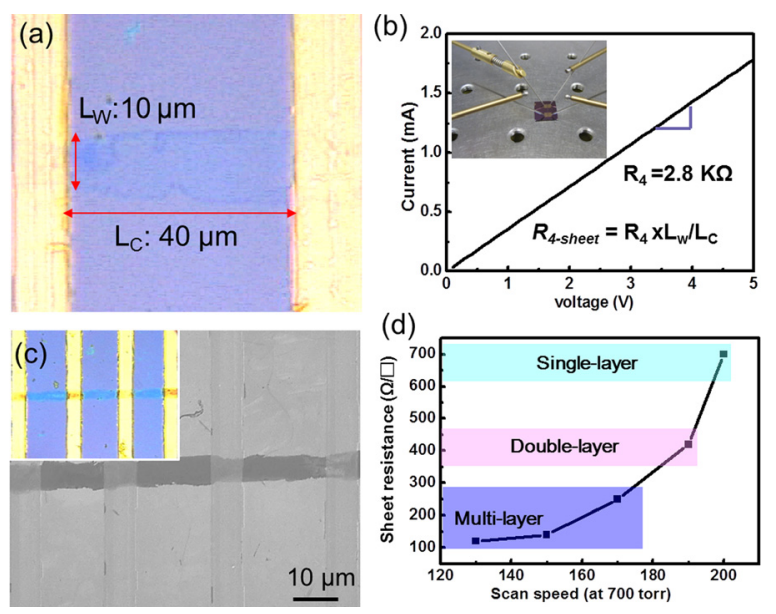

FIG. 4. (Color online) (a) Optical micrograph of transferred single-layer graphene on gold electrodes. (b) $I-V$ characteristic of single-layer graphene pattern. (c) SEM image of multi-layer graphene pattern. Inset shows an optical micrograph of multi-layer graphene pattern. (d) Sheet resistances of graphene pattern with respect to the scan speeds.

vapor-liquid-solid mechanism for the fabrication of graphene patterns was considered to explain the rapid growth of graphene patterns. We can control the number of graphene layers by tuning the laser scan speed on a nickel foil. Rapidly fabricated graphene patterns can be directly transferred to the desired position on prepatterned electrodes. Therefore, both the rapid graphene layer control and the position-controlled transfer techniques provide the controlled fabrication of transparent interconnections based on graphene patterns. We expect that the position-controlled transfer with the rapid single-step fabrication of graphene patterns provides an innovative pathway not only for graphene-based transparent interconnections but also for graphene-based devices.

This research work was financially supported by the National Science Foundation (CMMI 0758199 and CMMI 0852729), the Nebraska Research Initiative, and the Nebraska Center for Energy Science Research.

${ }^{1}$ F. Bonaccorso, Z. Sun, T. Hasan, and A. C. Ferrari, Nature Photon. 4, 611 (2010).

${ }^{2}$ C. Lee, X. Wei, J. W. Kysar, and J. Hone, Science 321, 385 (2008).

${ }^{3}$ A. K. Geim and K. S. Novoselov, Nature Mater. 6, 183 (2007).

${ }^{4}$ J. K. Wassei and R. B. Kaner, Mater. Today 13, 52 (2010).

${ }^{5}$ K. S. Kim, Y. Zhao, H. Jang, S. Y. Lee, J. M. Kim, K. S. Kim, J.-H. Ahn, P. Kim, J.-Y. Choi, and B. H. Hong, Nature 457, 706 (2009).

${ }^{6}$ X. Li, W. Cai, J. An, S. Kim, J. Nah, D. Yang, R. Piner, A. Velamakanni, I. Jung, E. Tutuc, S. K. Banerjee, L. Colombo, and R. R. Ruoff, Science 324, 1312 (2009).

${ }^{7}$ S. Bae, H. Kim, Y. Lee, X. Xu, J.-S. Park, Y. Zheng, J. Balakrishnan, T. Lei, H. R. Kim, Y. I. Song, Y.-J. Kim, K. S. Kim, B. Özyilmaz, J.-H. Ahn, B. H. Hong, and S. Iijima, Nature Nanotechnol. 5, 574 (2010).

${ }^{8}$ Y. Lee, S. Bae, H. Jang, S. Jang, S.-E. Zhu, S. H. Sim, Y. I. Song, B. H. Hong, and J.-H. Ahn, Nano Lett. 10, 490 (2010).

${ }^{9}$ X. Li, Y. Zhu, W. Cai, M. Borysiak, B. Han, D. Chen, R. D. Piner, L. Colombo, and R. S. Ruoff, Nano Lett. 9, 4359 (2009).

${ }^{10}$ Y.-C. Lin, C. Jin, J.-C. Lee, S.-F. Jen, K. Suenaga, and P.-W. Chiu, ACS Nano 5, 2362 (2011).

${ }^{11}$ J. B. Park, W. Xiong, Y. Gao, M. Qian, Z. Q. Xie, M. Mitchell, Y. S. Zhou, K. H. Han, L. Jiang, and Y. F. Lu, Appl. Phys. Lett. 98, 123109 (2011).

${ }^{12}$ M. A. Al-Nimr, Int. J. Thermophys. 18, 1257 (1997).

${ }^{13}$ J. Gavillet, A. Loiseau, C. Journet, F. Willaime, F. Ducastelle, and J.-C. Charlier, Phys. Rev. Lett. 87, 2755041 (2001).

${ }^{14}$ J. B. Park, S. H. Jeong, M. S. Jeong, J. Y. Kim, and B. K Cho, Carbon 46, 1369 (2008).

${ }^{15}$ P. Blakea, E. W. Hill, A. H. Castro Neto, K. S. Novoselov, D. Jiang, R. Yang, T. J. Booth, and A. K. Geim, Appl. Phys. Lett. 91, 063124 (2007). 